\title{
THE
}

\section{Analysis of Lagrangian Potential Vorticity Balance and Lateral Displacement of Water Parcels in Gulf Stream Meanders}

\author{
Tao Song \\ University of Rhode Island \\ Hans Thomas Rossby \\ University of Rhode Island, trossby@uri.edu
}

Follow this and additional works at: https://digitalcommons.uri.edu/gsofacpubs

\section{Citation/Publisher Attribution}

Song, T., \& Rossby, T. (1997). Analysis of Lagrangian Potential Vorticity Balance and Lateral Displacement of Water Parcels in Gulf Stream Meanders. J. Phys. Oceanogr., 27, 325-339. doi: 10.1175/ 1520-0485(1997)0272.0.CO;2.

Available at: https://doi.org/10.1175/1520-0485(1997)027<0325:AOLPVB>2.0.C0;2

This Article is brought to you for free and open access by the Graduate School of Oceanography at DigitalCommons@URI. It has been accepted for inclusion in Graduate School of Oceanography Faculty Publications by an authorized administrator of DigitalCommons@URI. For more information, please contact digitalcommons-group@uri.edu. 


\title{
Analysis of Lagrangian Potential Vorticity Balance and Lateral Displacement of Water Parcels in Gulf Stream Meanders
}

\author{
TaO SONG AND Tom Rossby \\ Graduate School of Oceanography, The University of Rhode Island, Kingston, Rhode Island
}

(Manuscript received 5 January 1995, in final form 30 July 1996)

\begin{abstract}
The balance of potential vorticity components following fluid parcel motion in Gulf Stream meanders was studied using RAFOS float data from the SYNOP Experiment. By introducing curvature dependent variations to the velocity and density fields, the authors relaxed the rigid field assumption used in earlier studies and examined closely 61 floats in the upper layers $\left(13^{\circ}-16^{\circ} \mathrm{C}\right)$ of the main thermocline. Float trajectories were segmented according to transition from crest to trough and trough to crest, and grouped by their positions relative to the current center. A total of 154 segments were collected to estimate the horizontal divergence and the mean lateral displacement of parcels under two distinct regimes: growing and decaying meanders.

Both spatial and temporal changes in curvature affect the regions of divergence in a meandering stream. On the one hand, horizontal divergence increases with increasing curvature magnitude, while on the other hand, the divergence pattern itself changes going from growing to decaying meanders. The growing amplitude meanders (i.e., cases where the magnitude of curvature increases in time) are found to be associated with divergence (convergence) upstream, and convergence (divergence) downstream of crests on the anticyclonic (cyclonic) side. This pattern is reversed for decaying meanders.

A parcel's cross-stream motion is found to be consistent with the pattern established earlier: upwelling/onshore from trough to crest and downwelling/offshore from crest to trough. When referenced to the locus of the velocity maximum, which itself is curvature dependent, the mean cross-stream displacements of parcels on the cyclonic and anticyclonic sides appear to be opposite in direction relative to the current center and hence result in difluence (confluence) up- (down) stream of crests for growing amplitude meanders and vice versa for decaying ones.

Cross-frontal fluid exchange is enhanced by changes in meander amplitude. The growth and decay of a meander are found to affect both the pathways and the intensity of fluid exchange. Comparisons of satellite IR imagery with RAFOS float trajectories suggest that the detraining of water associated with Gulf Stream meandering process occurs in both growing and decaying regimes.
\end{abstract}

\section{Introduction}

The dynamics of meandering jets have been the focus of much meteorological research for over half a century, with special interest in the jet stream and its role in the development of midlatitude weather systems. For example, between troughs and crests, ascending air masses, leading to condensation and precipitation, are due to low-level convergence and upperlevel divergence resulting from an adjustment of the cross-stream momentum balance of the meandering jet (Palmén and Newton 1969). As the jet stream begins to meander, a centrifugal force is induced. This forces an adjustment of the lateral pressure gradient, which, in turn, involves a mass redistribution to strengthen or

Corresponding author address: Dr. H. Thomas Rossby, Graduate School of Oceanography, University of Rhode Island, Kingston, RI 02881 . weaken the baroclinicity of the flow. The study of meandering jets in the ocean is more recent, in part due to the lack of observational techniques with which to conduct detailed studies of their kinematics. The first paper to highlight the similarities between the Gulf Stream and the atmospheric jet stream was published by Newton (1959), a study which he later updated at a Chapman conference on fronts (Newton 1978). The major points of those two papers, which depended heavily upon the hydrographic descriptions of the current that were available at the time, were 1) that both the jet stream and the Gulf Stream were to lowest order in geostrophic equilibrium, 2) their widths were controlled by the radius of deformation, and 3) that the structure of the currents was quite stable regardless of their instantaneous positions.

It is well known that any baroclinic flow that is not strictly geostrophic must have associated with it some form of vertical motion, which is the result of horizontal divergence or convergence. Thus, if one were able to 
measure the vertical velocity $w$, one would be able to construct a more complete picture of the kinematics of the flow as well as its dynamics and energetics. This accounts for the many efforts to estimate vertical velocities in the stream (Chew 1974; Bryden 1980; Hall 1986; Bane et al. 1989; Lindstrom and Watts 1994). A Lagrangian approach to the measurement problem became possible with the development of the isopycnal Swallow float by which vertical displacements could be observed directly (Rossby et al. 1985). The technique was specifically developed to permit studies of threedimensional particle motion in the Gulf Stream where the standard isobaric SOFAR floats had previously revealed substantial fluctuations in temperature due to vertical displacements along their horizontal trajectories (Shaw and Rossby 1984).

In 1984-85 a first experiment using the isopycnal RAFOS float technology to study fluid motion was conducted by sequentially releasing floats in the center of the stream from a commercial freighter that operates between Norfolk, Virginia, and Bermuda. The results of that study, which were reported in two papers (Bower and Rossby 1989, hereafter BR; Bower 1989), revealed a number of striking and important features about Lagrangian motion in the main thermocline of the Gulf Stream. The first is that a parcel's lateral motion in the stream has a pattern of upwelling (northward) as it flows from a trough to a crest, and vice versa from crests to troughs. This meander-induced cross-stream motion was shown to cause substantial cross-frontal exchange of fluid between the stream and the surrounding waters. The study showed that detrainment to the north always takes place upstream of meander crests and loss to the south between crests and troughs. It was also shown that loss from the stream was primarily due to these meander-induced motions rather than due to ring formation. From an analysis of the float tracks assuming conservation of potential vorticity (PV) and assuming the cross-stream structure of the down-stream velocity field was independent of curvature and downstream distance, Bower (1989) showed that there existed a systematic pattern of horizontal divergence: Divergence (convergence) was found upstream of crests (troughs) on the anticyclonic side, while convergence (divergence) was present on the cyclonic side. The analysis used to reach the above conclusions was constrained by the rigid field assumption. Specifically a cross-stream structure of downstream velocity that is independent of curvature was obtained from the results of a three-year PEGASUS study of the Gulf Stream (Halkin and Rossby 1985, hereafter HR).

In 1988-90 a major experimental study of the dynamics of the Gulf Stream known as SYNOP (SYNoptic Ocean Prediction) was undertaken. Its objective was to develop a greatly improved understanding of the dynamics of the Gulf Stream so that eventually a predictive capability for the meandering of the current could be developed. Building upon the experience from the earlier RAFOS float work a similar study was carried out, this time ballasting the floats for the upper thermocline or about $15^{\circ} \mathrm{C}$ where the downstream flow and potential vorticity (PV) gradient are much stronger than in the $9^{\circ}-12^{\circ} \mathrm{C}$ layers. In a first report of that experiment (Song et al. 1995, hereafter SRC), a detailed kinematical analysis of lateral displacements and pathways of escape from the stream revealed that the rate of loss of floats from the stream to both sides is much less on than on the deeper density layer. In this study, we shall focus on the dynamics of the float trajectories in terms of the Lagrangian potential vorticity balance. Given that the change in mean stratification across the stream is much larger on the $15^{\circ} \mathrm{C}$ than the $9^{\circ}-12^{\circ} \mathrm{C}$ surface, it would seem plausible that the meander divergence pattern established from the deeper layer floats will be more pronounced and perhaps more of its structure better resolved in both time and space. In the earlier (SRC) study, it was shown how escape from the stream depended not only upon the propagation of meanders, but also upon change in meander amplitude. Since divergence arises primarily from differential cross-stream motion of particles in the stream and particle motions are different between growing and decaying regimes, it is expected that a decaying meander could have significant impact on the divergence pattern established earlier.

To explore these issues and improve our understanding of the dynamics of fluid motion in the stream, the PV analysis methodology employed by Bower (1989) is applied to the 1988-90 RAFOS experiment. The approach is identical: A parcel's PV has, by scaling analysis, four major components: planetary, curvature, shear, and stretching vorticity. Of these the first two can be estimated directly, the third one is approximated using knowledge of where the float is within the current, and the last one is estimated on the assumption that PV is conserved on meander transit timescales. There is one technical difference: The frozen structure hypothesis is relaxed to allow for curvature dependent changes in the velocity and density structures. Information along subsurface float trajectories and from satellite imagery of sea surface temperature were used to identify how meander propagation, growth, and decay can affect the divergence/convergence patterns in the stream. The method for determining a growing or decaying meander regime and their different significance on the divergence pattern associated with meander are discussed in the next section. In section 3 , we will present details of the density and velocity model from which the shear vorticity is estimated. The technique for estimating curvature vorticity and the error analysis of the method are also included in the section. A case study is presented in section 4 and results of the analyses for the overall kinematical characteristics of the meandering stream are shown in section 5 . The extension of the analyses to include cross-frontal exchange 
processes is given in section 6 and followed by discussion in section 7 .

\section{Determination of growing and decaying meanders}

The unsteadiness of a meandering stream has two principal components: the propagation of phase and the change in amplitude in time. In order to investigate their role in the Lagrangian properties of a meandering stream, a better understanding of the kinematics of meanders is needed. Although the propagation, dispersion, and growth rate of meanders have been investigated through numerous studies (e.g., Watts and Johns 1982; Tracey and Watts 1986; Gilman 1988; Kontoyiannis and Watts 1994; Lee 1994), the effect of varying amplitude on meander dynamics is much less documented, in part because no means other than satellite IR imagery is more efficient in identifying the instantaneous growth or decay of a particular meander. And this can be made difficult, especially in winter when cloud coverage can be extensive and persistent. In this study, the float trajectories are sampled at 8-hour intervals (instead of the two-day composite IR imagery of SST). This provides a better temporal resolution about the meander state. A streamwise coordinate system is used throughout this study and is defined by downstream (parallel to the tangent of the streamline) and cross-stream/lateral (perpendicular to the streamline) directions with the origin marked by the center of the current. The current center, which is the locus of maximum downstream velocity, varies in depth along a given density surface (i.e., shifted laterally in terms of cross-stream distance) shoaling toward crests and deepening toward troughs (SRC). This information allows us to deduce the meander regime (i.e., growing or decaying amplitude) associated with each float passage.

The idea of determining growing versus decaying meanders is quite simple: If the lateral velocities of the current at adjacent crests, $v_{c}$ (crest) and troughs, $v_{c}$ (trough) have opposite sign, then the meander is judged to be growing when $v_{c}$ (crest) is northward and $v_{c}$ (trough) southward, or decaying if the sign is reversed, Fig. 1. In order to determine the lateral motion $v_{c}$ of the current center, the corresponding vertical motion $w_{c}$ needs to be determined first. Its estimation is based on an empirical expression relating the depth $P_{u \max }$ of the maximum downstream velocity and the curvature $\kappa$ of the meander section where the velocity maximum is observed (see Fig. 10 in SRC). The expression is obtained for the $13^{\circ}-16^{\circ} \mathrm{C}$ density surface (i.e., $26.6 \sim$ $\left.26.9 \sigma_{t}\right)$ and given in terms of path curvature $\kappa\left(\mathrm{km}^{-1}\right)$ :

$$
P_{u \max }=12000 \kappa+400
$$

so that the time rate of change in depth of the current center $w_{c}$ is approximated as
Steady amplitude


Decaying amplitude

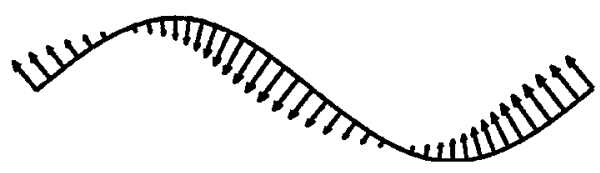

FIG. 1. A schematic view of the alongstream distributions of crossstream velocity $\left(v_{c}\right)$ of the current center for steady, growing, and decaying amplitude meanders. Arrows indicate the direction and relative magnitude of the cross-stream flow.

$$
w_{c}=\frac{\Delta P_{u \max }}{\Delta t},
$$

where $\Delta t$ is the sampling interval during which $P_{u \max }$ changes with $\kappa(s, t)$. Here $s$ and $t$ refer to the alongstream and time dependency of the path curvature. The corresponding cross-stream velocity $v_{c}$ of the velocity maximum can then be obtained from $w_{c}$, assuming that the isopycnal slope $S(\kappa)$ is known from observations (Halkin and Rossby 1985; Hummon 1995); that is,

$$
v_{c}=\frac{w_{c}}{S(\kappa)} .
$$

Analysis of CTD data from the Anatomy of Gulf Stream Meanders study (Hummon 1995) shows that the magnitude of the isopycnal slope in Gulf Stream meanders is on the order of $10 \mathrm{~m} \mathrm{~km}^{-1}$ (vertical vs cross-stream distance) and the value tends to be higher at troughs than crests. These are included in the modeled slope $S(k)$. Notice that for a zero-growth meander (i.e., $\partial \kappa / \partial t$ is zero in a frame moving at phase speed $c$ ), a change in $P_{u \max }$ will still be present between meander extrema due to the change in curvature along stream path $(\partial \kappa / \partial s \neq 0)$. This pure advection part of $v_{c}$ is on the order of $0.05 \mathrm{~m} \mathrm{~s}^{-1}$ and is expected to reach its maximum in the straight portion of the meander, for $\partial \kappa / \partial s$ is maximum there. It will vanish and 
therefore become increasingly smaller than the local part of $v_{c}$ (due to $\partial \kappa / \partial t$ ) at the point of minimum path curvature, that is, the peak (or maximum path curvature, i.e., the bottom) of crests (or troughs) for rapidly growing/decaying amplitude. With the RAFOS float dataset and using the results from SRC, this method thus provides a practical means to determine the growth or decay of a meander associated with each float passage. Although it is less sensitive to decaying meanders than growing ones due to the fact that the advective part of $v_{c}$ always has the sign opposite to the temporal part for decaying meanders, misinterpretations of a small number of weakly decaying meanders will have little statistical impact on the analysis.

In this study, 33 meanders were identified with decaying amplitude and 121 with growing ones (see section 5 below). A comparison with the satellite IR data (T. Lee 1994, personal communication) showed that of the 33 decaying meanders determined by float data, 13 are in good agreement, 18 are unavailable from IR data, and only 2 disagree marginally, whereas growing meanders have overwhelming consistencies between the two datasets. Note that the method used to determine a varying meander amplitude from the surface IR data was to digitize the surface northern edge of the stream, fit the digitized curve to a sine wave, and examine consecutively the change in amplitude of the fitted curves. Since the Gulf Stream meanders are usually not perfectly sinusoidal and the digitization of the north wall is somewhat subjective, the satellite method has significant uncertainties of its own.

\section{Estimation of potential vorticity components}

Following Bower (1989), the Lagrangian expression for potential vorticity in rotated stream coordinates defined as along (i.e., downstream) and normal to (i.e., cross stream) the streamline (such that the local vertical direction is perpendicular to the density surfaces) can be simplified by scaling analysis and is given as

$$
\frac{f+\kappa_{s} u_{s}+\partial u_{s} / \partial n}{h}=\text { const }
$$

where $f$ is the planetary vorticity; and $\kappa_{s} u_{s}$ and $\partial u_{s} / \partial n$ are curvature and shear vorticities in stream coordinates, respectively. The thickness of a hypothesized layer sandwiching the float is represented by $h$. It could just as well be a gradient $\left(\rho^{-1} \partial \rho / \partial z\right)^{-1}$. The magnitude of the term is arbitrary since it is not independent of the constant on the right-hand side. However, with $h$ representing a layer with a density difference of $0.2 \sigma$-unit or $O(100 \mathrm{~m})$, the constant on the right-hand side is about $10^{-6} \mathrm{~m}^{-1} \mathrm{~s}^{-1}$ as suggested by Leaman et al. (1989). While the technique for estimating each of the terms in Eq. (3.1) remains as in Bower (1989), the frozen field assumption is modified to include adjustments in velocity and density due to curvature.

\section{a. Planetary vorticity and layer thickness}

The planetary vorticity is the largest term in the numerator of Eq. (3.1). Together with the layer thickness it exerts a heavy constraint on how a fluid parcel can move about. It is estimated directly from the latitudinal position along the float track. On the other hand, there is no independent knowledge of the layer thickness $h$. It is, instead, estimated as a residual from a knowledge of all other terms including an assigned value to the constant on the right-hand side. This approach can be assured by the observational fact that downstream change in PV is very gradual.

\section{b. Shear vorticity}

Lateral shear in the Gulf Stream is an important component to the Lagrangian potential vorticity balance (LPVB). Its determination requires detailed knowledge of the velocity structure of the Gulf Stream, which can be estimated from a combination of PEGASUS (HR) and RAFOS float observations. In the earlier studies of LPVB in the lower thermocline $\left(9^{\circ}-12^{\circ} \mathrm{C}\right.$ ) (Bower 1989), the shear was estimated from the mean velocity field using both PEGASUS and float data. Inspection of the RAFOS float data (SRC) revealed no significant change in the lateral structure of the velocity field specifically with change in curvature. In the upper thermocline $\left(13^{\circ}-16^{\circ} \mathrm{C}\right)$ of the stream, on the other hand, the float data reveal a higher-order structure in the downstream velocity field that does change with curvature. This change is also accompanied by a lateral displacement of the maximum velocity core (SRC). Given this information, it is possible to modify the frozen field assumption to include variability due to changes in curvature of the current.

Using SYNOP float data and complemented with PEGASUS data at the edges, models representing the structure of temperature and velocity fields were constructed so as to include variations due to curvature. Two cross-stream profiles of downstream velocity were first constructed by fitting a cubic-spline to about 200 float observations at meander crests $\left(\kappa \sim-0.005 \mathrm{~km}^{-1}\right)$ and troughs $\left(\kappa \sim+0.005 \mathrm{~km}^{-1}\right)$, respectively (SRC). The higher-order structure (i.e., the hump on the anticyclonic side) is most pronounced at crests, Fig. 2. Velocities at the edges of the stream and for most of the cyclonic side where there are fewer float observations are based on the PEGASUS data, whereas float data are used for the central and anticyclonic parts of the stream. Since the PEGASUS data include no curvature information, it is only used as a complement to the float data. The underlying assumption is that not only the high velocity core but the whole velocity structure shifts laterally without much distortion or change along the edges of the current. For curvatures between -0.005 and +0.005 , the model velocity is simply a weighted average of the two profiles assuming a linear lateral dis- 




FIG. 2. Downstream velocity profile along density surfaces between $13^{\circ}$ and $16^{\circ} \mathrm{C}$. Model velocity is calculated as the weighted mean of velocities at crest and trough and indicated by solid curve for the case of zero curvature. Dashed curves represent the velocity profile at meander crest (marked by "o") and trough (marked by "+" for curvature of about $0.005 \mathrm{~km}^{-1}$ in magnitude.

placement of the velocity structure with curvature (Fig. 2 ). The weight itself is defined as a function of curvature $\kappa$ by

$$
\mathrm{r}=0.5-100 \kappa, \quad-0.005 \mathrm{~km}^{-1}<\kappa<0.005 \mathrm{~km}^{-1}
$$

so that for any given curvature $\kappa(s, t)$ within the range, the model velocity was obtained from the sum of the velocities weighted $r$ at crest and $(1-r)$ at trough and with the maximum velocity at $P_{u \max }$ defined in Eq. (2.1). For other values of $\kappa$ (about $30 \%$ of cases), the crest $\left(\kappa=-0.005 \mathrm{~km}^{-1}\right)$ or trough $\left(\kappa=0.005 \mathrm{~km}^{-1}\right)$ velocity profiles are used as approximations. This weighted average procedure allows a smooth transition of the velocity structure between meander extrema as observed previously (SRC). The shear vorticity term was estimated by taking the derivative with respect to depth change (measured in dbar or equivalently in meters) along an isopycnal surface and multiplying by the slope of the isotherm (which is a very good approximation to the isopycnal slope) to obtain the lateral shear along that surface. The local isothermal slope was determined from a modified empirical model of the cross-stream temperature structure (see appendix in BR). In the present study, the scale widths of the current used in the model were modified and parameterized with temperature and curvature based on the analysis of different data sets, that is, CTDs and XBTs (Hummon 1995). This means that the curvature effect is included with respect to both the velocity structure and the cross-stream thermal (or density) field. However, tests indicated that the effect on the latter is small, meaning that the relaxation to the rigid field comes essentially from the velocity (Fig. 2) than from the temperature (or density) field.

\section{c. Curvature vorticity}

The curvature vorticity is defined as the product of the downstream velocity $u_{s}$ and the curvature of the stream $\kappa_{s}$. The method for estimating the curvature is the same as the one used in the Bower study (1989); that is, trajectory curvature $\kappa_{t}$ was first computed following the float track and then converted into stream path curvature $\kappa_{\mathrm{s}}$ according to

$$
\kappa_{s}=\kappa_{t} \frac{V}{V-c \cos (\alpha)},
$$

where $V$ is particle speed, $c$ is propagation velocity of a meander, and $\alpha$ is the angle between the directions of flow and phase propagation (Holton 1979). In this study, a typical value of $0.08 \mathrm{~m} \mathrm{~s}^{-1}$ for $c \cos (\alpha)$ was used for Gulf Stream meanders (Gilman 1988). This conversion is necessary since trajectory is different from a streamline for unsteady motion. One can view Eq. (3.3) as a statement of Doppler correction.

The transformation of horizontal velocity from geographical to curvature-dependent stream coordinates requires an extra step because the downstream component $u_{s}$ must be deduced from a parcel's cross-stream velocity $v_{s}$ relative to the center of the stream [see Eq. (3.6)]. In this study, we define the center by the locus of downstream velocity maximum for two reasons. First, it is the point where shear vorticity changes sign such that the stream can be subdivided into a cyclonic and an anticyclonic sides. Second, it is a definition consistent with the earlier study conducted under the rigid field assumption (Bower 1989), permitting direct comparisons with it. Since, by definition, the center is curvature dependent, lateral (or vertical) motion can be anticipated. It is convenient to partition the lateral (or vertical) motion $v_{f}$ (or $w_{f}$ ) seen by a float into two components: the motion of a float (or fluid parcel) $v_{s}$ relative to the center (subscript $s$ refers to stream coordinates) and the lateral motion of the center $v_{c}$ along a density surface; that is,

$$
\boldsymbol{v}_{f}=\boldsymbol{v}_{s}+\boldsymbol{v}_{c}
$$

A schematic view of a parcel's relative lateral displacement is given in Fig. 3 where the time rate of change in $\Delta x_{\text {float }}$ (or $\Delta z_{\text {float }}$ ) and $\Delta x_{\text {current }}$ [or $\Delta z_{\text {current }}, \Delta z_{\text {current }}$ here is equivalent to $\Delta P_{u \max }$ in Eq. (2.2)] can yield $v_{f}$ (or $w_{f}$ ) and $\boldsymbol{v}_{c}$ (or $w_{c}$ ), respectively. In this study, the velocity $\boldsymbol{v}_{c}$ is estimated from $w_{c}$ using Eq. (2.3) and the quantity $v_{f}$, the total cross-stream velocity component seen by a float, is determined in a similar manner as for $v_{c}$ :

$$
v_{f}=\frac{w_{f}}{S(\kappa)},
$$




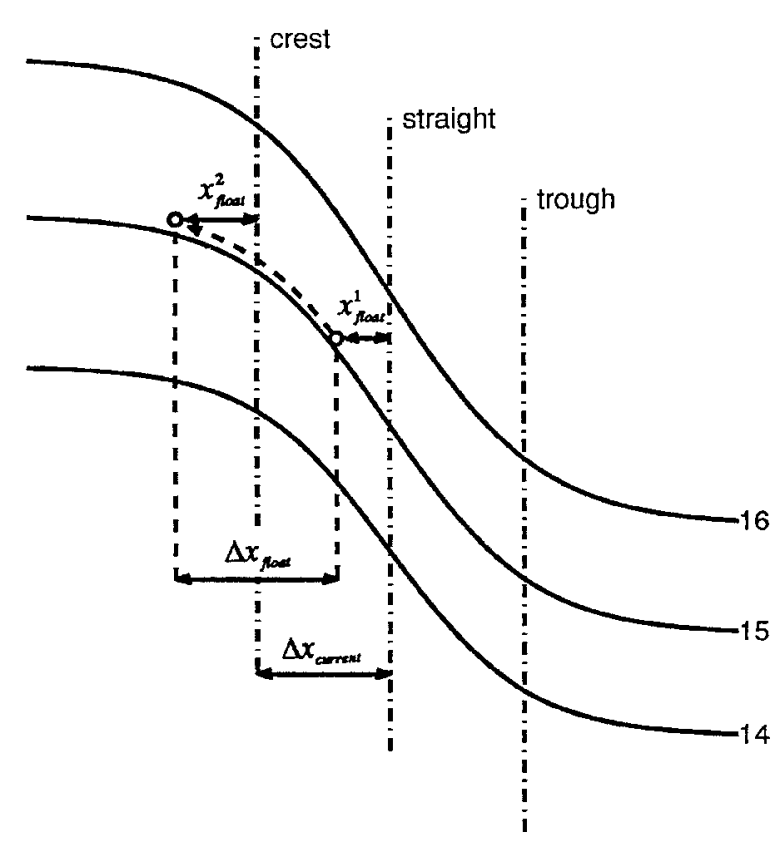

FIG. 3. A schematic view of a parcel's lateral displacement relative to the velocity maximum. Dash-dotted lines indicate the locus of velocity maximum (i.e., the center) relative to $15^{\circ} \mathrm{C}$ density surface at the crest $(\kappa<0)$, trough $(\kappa>0)$, and straight $(\kappa=0)$ portions of a meander, respectively. The change in cross-stream position of a float relative to the current center is given by $x_{\text {float }}^{2}-x_{\text {float }}^{1}=\Delta x_{\text {float }}-$ $\Delta x_{\text {current }}$.

knowing that $w_{f}$ is identical to $w$ in geographical coordinates. Therefore, $v_{s}$ is known from (3.4) by subtracting $v_{c}$ from $v_{f}$. A similar procedure can be applied to obtain $w_{s}$ as $w_{f}=w_{s}+w_{c}$. Finally, the downstream component $\left(u_{s}\right)$ will be deduced using the vector invariance property; that is,

$$
u_{s}^{2}+v_{s}^{2}+w_{s}^{2}=u^{2}+v^{2}+w^{2}=V^{2},
$$

where $u, v$, and $w$ are float velocity components in geographical coordinates.

\section{d. Error estimates}

The errors associated with each vorticity component in Eq. (3.1) were discussed in detail by Bower (1989). Most arguments remain valid since the same methodology is being applied to a similar dataset.

Planetary vorticity: The error in planetary vorticity $f$ is mainly associated with the uncertainty in determining float positions. Assuming a linear variation of $f$, that is, a $\beta$ plane where $\beta=2 \times 10^{-11} \mathrm{~m}^{-1} \mathrm{~s}^{-1}$ and a pointto-point error of $\pm 2 \sim 4 \mathrm{~km}$ in float position, the uncertainty in $f$ is about $\pm(0.05$ to 0.10$) \times 10^{-6} \mathrm{~s}^{-1}$ or $\pm(0.05$ to 0.10$) \%$ of $f$.

Curvature vorticity: Since a float velocity is determined by the time rate of change of float position using a simple centered-difference scheme, its uncertainty due to uncorrelated position errors, is found to be

$$
\frac{\sqrt{2} \times \Delta x}{2 \times \Delta t}=\frac{\sqrt{2} \times(2 \sim 4) \mathrm{km}}{2 \times 8 \text { hours }} \approx \pm 0.10 \mathrm{~m} \mathrm{~s}^{-1} .
$$

If we also assume that the uncertainties are $\pm 0.12 \mathrm{~m}$ $\mathrm{s}^{-1}$ for phase velocity $c$ (Gilman 1988) and $\pm 0.001 \mathrm{~km}^{-1}$ for trajectory curvature $\kappa_{t}$, a standard propagation of these errors would lead to uncertainties of \pm 0.0022 $\mathrm{km}^{-1}$ for $\kappa_{s}$ according to (3.3) and, hence, $\pm 2.5 \times 10^{-6}$ $\mathrm{s}^{-1}$ or about $\pm 3 \%$ of $f$ for $\kappa_{s} u_{s}$. The above estimates are based on typical values of $1.0 \mathrm{~m} \mathrm{~s}^{-1}$ for $u_{s}, 0.08 \mathrm{~m} \mathrm{~s}^{-1}$ for $c$, and $0.01 \mathrm{~km}^{-1}$ for both $\kappa_{t}$ and $\kappa_{s}$. If a smaller value of $u_{s}$, say $0.5 \mathrm{~m} \mathrm{~s}^{-1}$, were used, the uncertainties would be $0.0092 \mathrm{~km}^{-1}$ for $\kappa_{s}$ or less than $10 \%$ of $f$ for $\kappa_{s} u_{s}$. This reflects the situation where a float is away from the current center, and we found that in less than $4 \%$ of cases a float has velocity smaller than $0.5 \mathrm{~m} \mathrm{~s}^{-1}$.

Shear vorticity: The shear vorticity term $\partial u_{s} / \partial n$ is approximated by the first difference of the model velocity at the appropriate cross-stream position (i.e., depth of the desired isopycnal). The model velocities have been found to be in good agreement with both float and PEGASUS data to within $\pm 0.20 \mathrm{~m} \mathrm{~s}^{-1}$ for the part of the stream where float observations are most frequent. The shear vorticity from our model was compared to the one from the rigid model for each of the 61 floats. We found that there are only about $12 \%$ of the cases where the differences in shear vorticity between the two models exceed $10 \%$ of $f_{o}$ due to large meander amplitude $(|\kappa|$ $\left.>0.005 \mathrm{~km}^{-1}\right)$. On the other hand, the shear vorticity estimates from both models are quite consistent for small meanders $\left(|\kappa|<0.001 \mathrm{~km}^{-1}\right)$. The relaxed model was also compared to the mean PEGASUS data and the difference in shear is about $\pm(10 \sim 15) \times 10^{-6} \mathrm{~s}^{-1}$. A large fraction of this difference is due to the fact that shear vorticity from the model varies with path curvature, while this curvature dependence is barely reflected in the PEGASUS mean section, although on average the Gulf Stream mean path appears to have a weak anticyclonic curvature $\left(-0.001 \mathrm{~km}^{-1}\right.$ or larger) near $73^{\circ} \mathrm{W}$. Taking this fact into account, we judge that the error in $\partial u_{s} / \partial n$ is limited to $(5 \sim 10) \times 10^{-6} \mathrm{~s}^{-1}$.

\section{A case study}

To illustrate in detail the balance of potential vorticity components following a float, we examine the changes of each component along its trajectory. Consider float 192 , launched near $73^{\circ} \mathrm{W}$ on the anticyclonic side of the stream on 1 June 1989: Its 45-day trajectory (Fig. 4a) shows three major meanders. The corresponding temperature and pressure changes, Figs. $4 \mathrm{~b}$ and $4 \mathrm{c}$, indicate good isopycnal behavior: $\Delta T \leq 0.6^{\circ} \mathrm{C}$ between a pressure range of $375-600$ dbars. Figure $4 d$ shows the crossstream position $\left(x_{\text {float }}\right)$ of the float relative to the current center. This quantity is defined in Fig. 3 and determined according to 

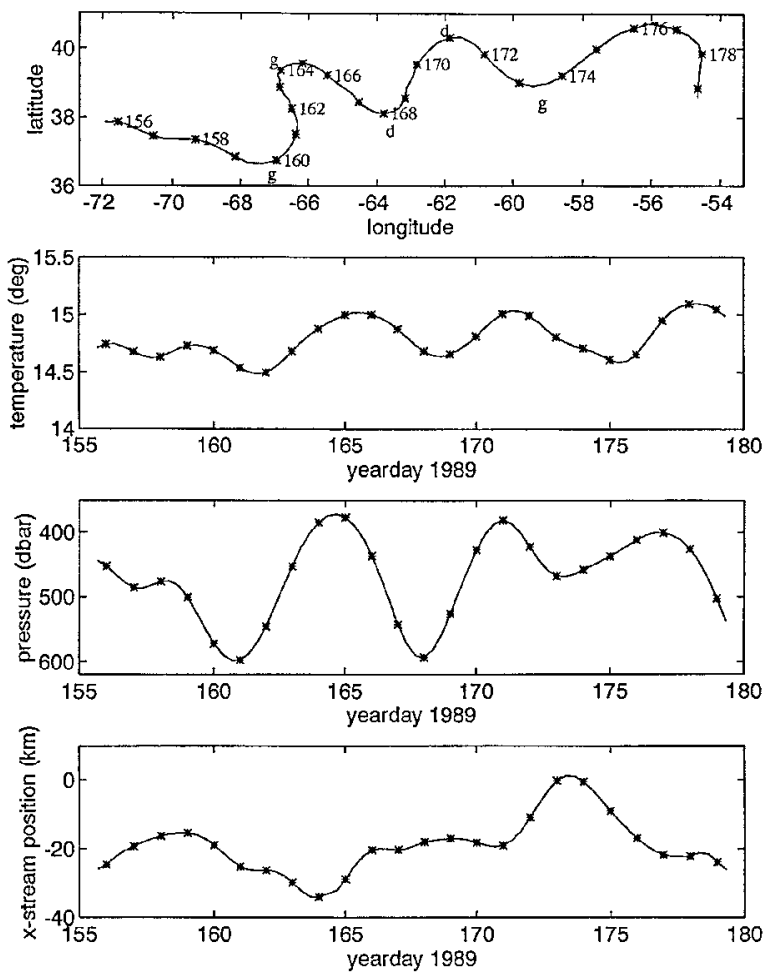

FIG. 4. (a) Trajectory, (b) temperature, (c) pressure records, and (d) cross-stream position relative to the velocity maximum of float 192. Numbers and small letters along the track indicate year day, growing $(\mathrm{g})$ and decaying (d) meander crests or troughs. Stars are marked on a daily basis. The order of the panels in this and following figures is left-to-right and top-to-bottom.

$$
x_{\text {float }}=\frac{P_{\text {float }}-P_{u \max }}{S(\kappa)},
$$

where $P_{u \max }$ is defined in (2.1) and $P_{\text {float }}$ is the depth (or pressure in dbar) of a float on an isopycnal surface. Although the amplitude of the meander is fairly large, $O(100 \mathrm{~km})$, as seen from its trajectory, the rms lateral displacement of the float relative to the current is only about $\pm 7.6 \mathrm{~km}$ at a mean position of $18.6 \mathrm{~km}$ south of the velocity maximum. The largest lateral excursion in its lifetime took place between year days 164 and 174 . During this period, the float transited from a growing meander crest ( $\sim$ day 164$)$ through a decaying meander trough ( $\sim$ day 168$)$, then from a decaying crest $(\sim$ day $171)$ to a growing trough ( $\sim$ day 173 in Fig. $4 a)$. The weak lateral displacement in the presence of large meanders (day 165 170) was identified by superimposing float track onto imagery of the SST field. The subsurface float trajectory showed little variation in cross-stream position relative to the sea surface north wall, consistent with the result showing in Fig. 4d from the relaxed model. Further, before day 168, the float moved toward the current center as it was transiting from a crest to a trough (days 156 159 and 164 168) and toward the southern edge after it reached the trough (day 160) (Fig. 2b). Between day 164 and 171, the float was transiting from a growing crest (day 164) to a decaying trough (day 168) and crest (day 171). The corresponding lateral displacement especially between days 166 and 171 was very small, probably because of the two competing effects from temporal and spatial change in curvature on a parcel's cross-stream motion (see sections 5 and 6). In terms of Eq. (2.5), this means that the dynamical adjustment during this particular period of time and space is taking place such that a parcel's motion $v_{f}$ is essentially the same as that of the current's center $v_{c}$, rendering $v_{s}$ very small. After day 173 , the meander resumed to its growing regime as well as the pattern of lateral displacement as indicated in Fig. 4d between day 171 and 177, implying that $v_{c}$ is smaller in magnitude than $v_{f}$. This more complicated lateral displacement pattern is striking and apparently different from that of the rigid model (BR), which suggests that parcels move to the left from trough to crest and to the right from crest to trough when facing downstream. The difference is due to the fact that lateral motion in this study is measured relative to the maximum velocity core, while in $\mathrm{BR}$ it is relative to a fixed position on a given density surface. It should not be understood as an inconsistency because a parcel's southward displacement (i.e., to the right) in a growing amplitude meander on the anticyclonic side between trough and crest reflects that the locus of the velocity maximum is shoaling faster than a parcel situated farther offshore does (i.e., $v_{c}>v_{f}$ ). In the earlier studies, the complicated aspect of lateral displacement was simply masked by the rigid field assumption, which did not allow for the current structure to move laterally. As a consequence patterns of a parcel's lateral motion (i.e., $v_{s}$ ) can only be distinguished between upstream and downstream of crest (or trough) but not between cyclonic and anticyclonic sides. The connection of this more detailed pattern of lateral motion with the dynamics of the meandering stream will become clear as we proceed with the analysis of the float data (sections 5 and 6).

The downstream and cross-stream velocity components of float 192 are shown in Fig. 5. As discussed in section 2, a parcel's cross-stream velocity can be expressed as the sum of its motion related to the velocity maximum and the shoaling/deepening of the velocity structure. The magnitude of a parcel's cross-stream motion as well as its pattern is therefore significantly affected by the relaxation of the rigid assumption. The cross-stream flow appears to be much weaker when referenced to the lateral displacement of the velocity maximum, Fig. 5b. Since the downstream velocity is an order of magnitude higher than the cross-stream one, only small changes were observed with or without this relaxation, Fig. 5a.

A careful examination of the LPVB for float 192 will be helpful in illustrating the impact of unsteady meanders on a parcel's cross-stream motion. The four com- 

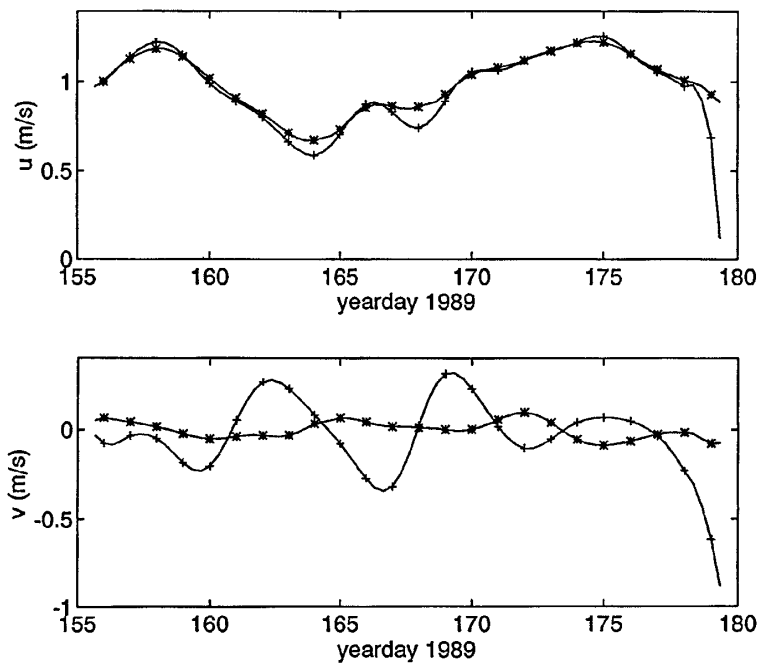

FIG. 5. (a) Downstream and (b) cross-stream velocity components of float 192 in natural coordinates referenced to a fixed point on isopycnal surface $(+)$ or to the locus of downstream velocity maximum (*).

ponents defined in Eq. (3.1) can be written in a nondimensional form to the first-order approximation:

$$
\frac{f+\kappa_{s} u_{s}+\partial u_{s} / \partial n}{h} \frac{h_{o}}{f_{o}}=\frac{f}{f_{o}}+\frac{\kappa_{s} u_{s}}{f_{o}}+\frac{1}{f_{o}} \frac{\partial u_{s}}{\partial n}-\frac{f}{f_{o}} \frac{\Delta h}{h_{o}},
$$

where $f_{o}$ is the planetary vorticity at the central latitude of a float track and $h_{\mathrm{o}}$ and $\Delta h$ are the mean and the deviation from the mean layer thickness along a float track. Each of the terms on the right-hand side was estimated for float 192, Fig. 6. Planetary vorticity calculated from the float latitudinal position varies between 8.7 and $9.5 \times 10^{-5} \mathrm{~s}^{-1}$ or about $10 \%$ of $f_{o}$, Fig. 6a. Changes in curvature vorticity, Fig. 6b, show the variations primarily due to change in curvature: maxima at troughs and minima at crests.

The shear vorticity, Fig. 6c, is always negative since the float was launched on the anticyclonic side and never reached the center of the stream (except near day 173). We should emphasize that when estimating shear vorticity account was taken for the lateral sliding of the velocity field relative to the density surface and, hence, distortions of the velocity structure due to changes in curvature were in a statistical sense included. Between day 166 and 170, the change in cross-stream position is very small, Fig. 4b, whereas the change in shear vorticity is significant, Fig. 6c. It is the estimated lateral displacement of the maximum velocity core and the corresponding structural change that causes the change in shear without being much displaced relative to the current center. Here we should point out that although the parcel marked by float 192 has weak or no lateral motion relative to the current center, other parcels across the stream may still have significant lateral displace-
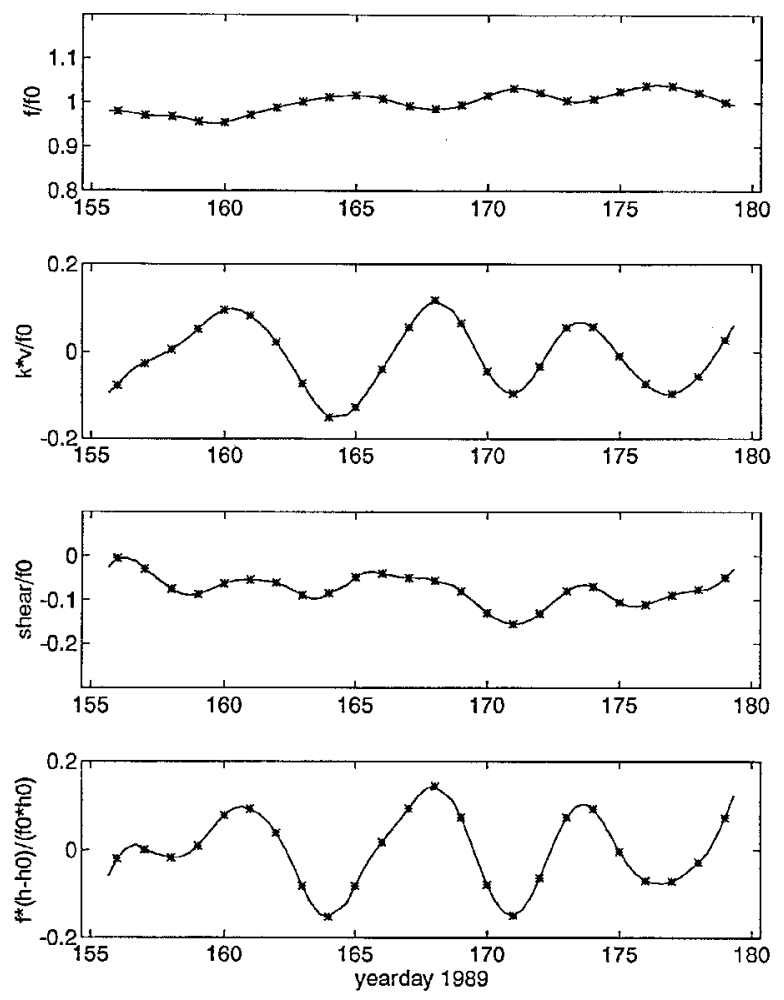

FIG. 6. Variations of (a) planetary, (b) curvature, (c) shear vorticity components, and (d) layer thickness along the trajectory of float 192. All quantities are nondimensionalized by $f_{o}$ of the planetary vorticity at the central latitude of the float track and $h_{o}$ of the mean layer thickness along the float track.

ments and hence cause important changes in shear. Note that during this period, float 192 was transiting a decaying amplitude meander and therefore subject to the two competing effects of temporal (decaying amplitude) and spatial (along float track) curvature variations. This is also reflected in Fig. 5b where cross-stream motion relative to the current is almost zero.

The uncertainty in the shear vorticity estimates can lead to apparent inconsistencies as happened near day 173 when the shear vorticity is about $-0.75 \times 10^{-5} \mathrm{~s}^{-1}$ ( $\sim 10 \%$ less than $f_{o}$ in Fig. $6 \mathrm{c}$ ) yet the float appears to be at the center (Figs. 4d and 5a). This is because shear and cross-stream position are estimated independently. Given the float pressure $\left(P_{\text {float }}\right)$, the shear is approximated by taking the first-order difference of the model velocity (Fig. 2), whereas the position is obtained from Eq. (4.1). The error is within the estimated uncertainty in shear $\pm 0.5 \times 10^{-5} \mathrm{~s}^{-1}$ and position $\pm 2 \mathrm{~km}$. Examination of 61 floats shows that occurrence of this error is not often (less than 7\%) and only slightly increases the noise level of the ensemble analysis.

The pattern of variation in layer thickness, Fig. 6d, is consistent with Fig. 13 of Bower (1989) for floats on the anticyclonic side: Stretching takes place from crest to trough and conversely from trough to crest, closely 
following the variation of curvature vorticity. In this particular case, the shear vorticity change modulates the magnitude of the layer thickness variation while curvature vorticity dominates the patterns. The mean thickness of the water column is $81 \mathrm{~m}$ with a standard deviation of $\pm 6 \mathrm{~m}$. Maximum change, being about $30 \%$ of the mean, occurred between yeardays 168 and 171, the period when the float transited from a decaying meander trough to the next decaying crest (Fig. 4a).

\section{Divergence and convergence patterns in Gulf Stream meanders}

To study and systematize the divergence and convergence patterns in Gulf Stream meanders, we applied the above analysis technique to 61 RAFOS floats in the $13^{\circ}-16^{\circ} \mathrm{C}$ layers. The absolute vorticity and the four components of potential vorticity were estimated along each float track by breaking it into sections of crest-totrough and trough-to-crest so that divergence estimate was made on a segmental basis. In an attempt to clarify the effect of the unsteady meanders, specifically the growth/decay of meander amplitude rather than the propagation of the phase, all segments were grouped into two classes, namely, growing and decaying amplitude meanders. Further, following Bower's analysis (1989) we consider the following subgroups: (a) trough to crest cyclonic; (b) crest to trough cyclonic; (c) trough to crest anticyclonic; and (d) crest to trough anticyclonic side; a total of 8 groups being examined. Of the 61 float trajectories investigated, we were able to document 154 segments with 121 (79\%) for growing meanders and 33 (21\%) for decaying ones, a percentage consistent with that of IR image analysis (T. Lee 1994, personal communication). The groups on the anticyclonic side of the stream are much more heavily sampled than those of the cyclonic side, and one group, the crest-to-trough cyclonic group in the growing meander class, had no data at all.

The divergence associated with each segment was estimated by first computing the relative substantial change of absolute vorticity at each sampling interval according to

$$
\frac{\partial w}{\partial z}=\frac{1}{\zeta_{a}} \frac{d \zeta_{a}}{d t} \approx \frac{1}{\frac{1}{2}\left(\zeta_{a 1}+\zeta_{a 2}\right)} \frac{\zeta_{a 2}-\zeta_{a 1}}{\Delta t},
$$

where $\zeta_{a}$ is the absolute vorticity, indices 1 and 2 refer to the beginning and the end of the interval, and $\Delta t$ is the sampling period. These values were then averaged by the number of samples over the whole segment to represent the divergence estimate associated. Figure 7 shows these estimates as a function of curvature for the growing amplitude meanders. The results are displayed in four panels corresponding to the four subgroups defined above. Bars associated with each point are standard deviations from the mean, larger for some and smaller for others, reflecting the scattering of the estimates over that particular segment. As indicated in Fig. 7 , the divergence and convergence patterns for growing meanders are quite robust and consistent with Bower's analysis (1989) for the $9^{\circ}-12^{\circ} \mathrm{C}$ layers of the main thermocline. The overall divergence estimate (the weighted mean) as well as the standard deviation and standard error of each group is given in Table 1a. Except the crest-to-trough cyclonic group where no data were available, all estimates are significantly different from zero at or above $90 \%$ confidence level. Similarly, the results for decaying amplitude meander class are given in Fig. 8 , where a striking feature emerges by comparing it with Fig. 7. The divergence pattern turns out to be exactly opposite to that of the growing meander class, that is, wherever the divergence is in the growing meander regime, the convergence appears in the decaying meander class and vice versa. Although only 33 segments were available for this group, we believe that the pattern is real, for the estimates of divergence in each group were found to be significantly different from zero at $71 \%$ $99 \%$ confidence levels (Table $1 \mathrm{~b}$ ).

Notice that the magnitude of the divergence is also curvature dependent. As shown in Figs. 7c,d and Figs. $8 \mathrm{~b}, \mathrm{c}$, the correlation between divergence and curvature is considerable, especially in the two largest groups of 55 and 62 estimates where divergence/convergence increases with the increase of curvature magnitude. In other words, for a given regime, larger meander amplitude (i.e., smaller radius of curvature for a given wavelength) will generally lead to stronger cross-stream motions. These are in turn manifested as larger horizontal divergence (or convergence) and must be due to nonuniform lateral displacements (the alongstream variations of downstream velocity are weaker than the lateral ones). However, exceptions may be found at the transition stage where competing effects between spatial (alongfloat track) and temporal (decaying amplitude) changes in curvature may weaken or even cancel the cross-stream motion. The paucity of data on the cyclonic side almost certainly reflects the fact that most floats were launched on the anticyclonic side and the sampling was therefore unevenly distributed across the stream.

\section{Lateral displacement and its relation to fluid exchange with surrounding waters}

The cross-stream dynamical balance in Gulf Stream meanders is achieved through continual lateral adjustments. This was observed from the lateral displacement of fluid parcels relative to the current center. Dividing the crest-to-trough or trough-to-crest segments into the eight groups defined previously, we calculated the mean lateral displacement of floats at eight hour intervals in each group. For growing amplitude meanders, the mean lateral displacements of parcels relative to the current center (defined by the maximum velocity core) are found to be positive on the cyclonic and negative on the an- 

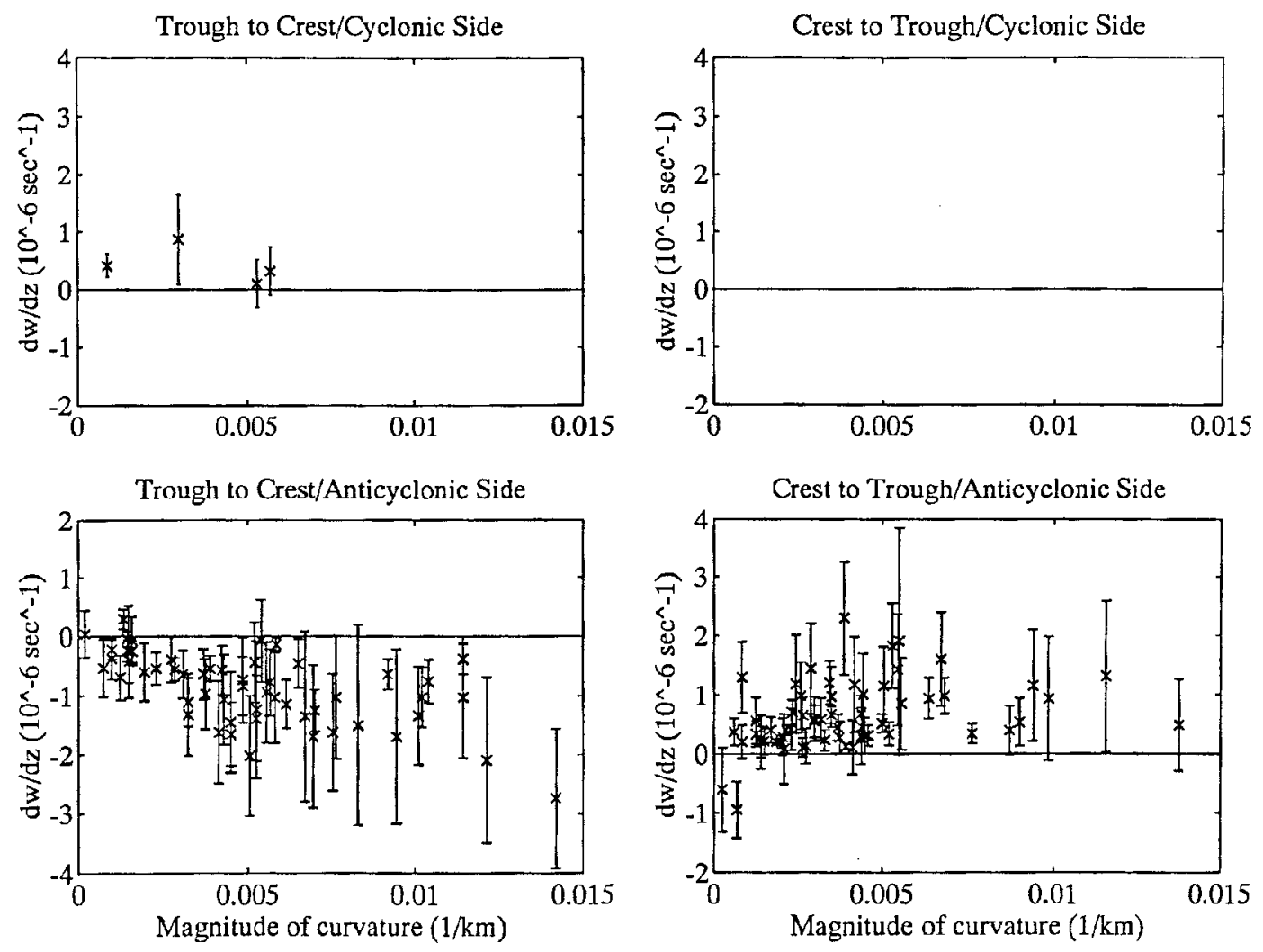

FIG. 7. Horizontal divergence estimates in terms of curvature change for growing amplitude meanders. All units are in $10^{-6} \mathrm{~s}^{-1}$.

ticyclonic side upstream of meander crests, that is, moving away from the current center and resulting in difluence along density surfaces in trough-to-crest segments, Fig. 9. Similarly, confluence is demonstrated by the

TABLE 1. Estimates of horizontal divergence in Gulf Stream meanders $\left(13^{\circ}-16^{\circ} \mathrm{C}\right.$ layers). Values in each column are weighted mean, weighted standard deviation, weighted standard error, number of observations, and the confidence level at which the mean is different from zero.

\begin{tabular}{|c|c|c|c|c|}
\hline \multirow[b]{2}{*}{$\begin{array}{l}\text { Statis- } \\
\text { tics }\end{array}$} & \multicolumn{2}{|c|}{$\begin{array}{l}\text { Trough to crest } \\
\qquad\left(10^{-6} \mathrm{~s}^{-1}\right)\end{array}$} & \multicolumn{2}{|c|}{$\begin{array}{l}\text { Crest to trough } \\
\quad\left(10^{-6} \mathrm{~s}^{-1}\right)\end{array}$} \\
\hline & Cyclonic & $\begin{array}{l}\text { Anti- } \\
\text { cyclonic }\end{array}$ & Cyclonic & $\begin{array}{l}\text { Anti- } \\
\text { cyclonic }\end{array}$ \\
\hline \multicolumn{5}{|c|}{ (a) Growing amplitude meanders } \\
\hline Mean & -0.370 & 0.359 & & -0.290 \\
\hline Std dev & 0.176 & 0.410 & & 0.222 \\
\hline Std error & 0.162 & 0.044 & & 0.023 \\
\hline$n$ & 4 & 55 & & 62 \\
\hline Conf level & $90 \%$ & $99 \%$ & & $99 \%$ \\
\hline \multicolumn{5}{|c|}{ (b) Decaying amplitude meanders } \\
\hline Mean & 0.183 & -0.147 & -0.543 & 0.235 \\
\hline Std dev & 0.236 & 0.182 & 0.486 & 0.242 \\
\hline Std error & 0.107 & 0.063 & 0.087 & 0.182 \\
\hline$n$ & 3 & 8 & 17 & 5 \\
\hline Conf level & $75 \%$ & $95 \%$ & $99 \%$ & $71 \%$ \\
\hline
\end{tabular}

mean lateral displacements on the crest-to-trough section of the stream. As expected, this pattern is reversed when meander regime switches from growing to decaying phase, Fig. 10. The uncertainties of our estimates were assessed for each group and their associated confidence levels are found to be at $95 \%$ or higher, Table 2. A schematic view of parcels' mean lateral displacements and regions of divergence/convergence in Gulf Stream meanders are given in Fig. 11. Note that for growing meander case, the crest-to-trough cyclonic side quadrant shows the expected result in a situation from which data in that group were available.

A direct consequence of a parcel's lateral displacement is the concomitant exchange between the Gulf Stream and surrounding waters. To understand how this takes place, we examined all float escapes from the stream due to meandering and compared them case by case with the patterns of lateral motion discussed above. Float loss was observed occurring in both growing and decaying meanders, but only on those sides where mean lateral displacements were outward. Of the 14 floats lost due to meandering, 12 are confirmed to be in agreement with the above discussion. The other two are unknown due to insufficient information.

Float escapes were often associated with rapid lateral 



FIG. 8. As in Fig. 7 but for decaying meanders.

displacements. The rms cross-stream velocity during the period when a float transited the last crest-to-trough or trough-to-crest segment just prior to leaving the stream is about $0.14 \mathrm{~m} \mathrm{~s}^{-1}$, contrasting to the rms value of 0.06 $\mathrm{m} \mathrm{s}^{-1}$ estimated from those same floats but in the segments a few days earlier. In terms of potential vorticity components, conspicuous difference between escaping and nonescaping floats was only observed in the stretching (or squeezing) of the water column, consistent with the large lateral displacement relative to the stream center.

\section{Discussion}

One aspect of fluid motion in a meandering stream, namely, the mean lateral displacement of a parcel and the pattern of horizontal divergence, was examined in terms of growing and decaying meanders. With the relaxation of the rigid field assumption, the current center defined by the maximum downstream velocity was allowed to shift laterally as a function of the path curvature. Although such a relaxation sounds simple, the pattern of lateral motion of fluid parcels reveals more structure when referenced to this curvature-dependent stream coordinate system. The mean lateral displacements on both sides tend toward (away from) the center as they proceed from crests (troughs) to troughs (crests) during the growing stage of a meander (Table $2 \mathrm{a}$ and Fig. 11a). As a consequence, difluence was statistically observed upstream and confluence downstream of crests along a constant density surface. For decaying meanders, the pattern is reversed and show in Table $2 b$ and Fig. 11b. At first sight, this pattern of lateral motion is different from that of BR. A closer look indicates that this is just another statement of the same pattern viewed from a laterally moving reference frame. Generally speaking, the lateral shift of the maximum velocity core $\left[O\left(0.05 \mathrm{~m} \mathrm{~s}^{-1}\right), \mathrm{SRC}\right]$ to which parcels are referenced is different from that of parcels themselves. Parcels at different cross-stream position may appear moving toward or away from the center depending on their speed relative to the maximum velocity core. With the rigid field assumption, this relative motion is not seen because the center of the stream was forced to be fixed relative to the density field by attributing all lateral motion to the parcels. In that reference frame, all parcels move in the same direction although not necessarily at the same speed, yielding a simple pattern of upwelling onshore from trough to crest and downwelling offshore from crest to trough. The new pattern actually reflects the same cross-stream motion as the earlier one but relative to a velocity field that shifts laterally according to curvature.

The effect of curvature change on a parcel's lateral 



FIG. 9. Mean lateral displacements of parcels in growing amplitude meanders. Units are in kilometers.

displacement can be viewed in two ways. First, the change in curvature along a trajectory requires instantaneous adjustment of the cross-stream momentum balance through lateral motion. A larger meander amplitude means a greater variation in curvature along the stream path for a given wavelength, therefore inducing stronger lateral motions. Second, a temporal increase in curvature magnitude often indicates the growth of a meander and hence causes lateral displacements of parcels for adjustment. The faster the temporal change, the stronger the response of the velocity field. In other words, the unsteadiness of meander amplitude plays a crucial role in determining both the sign and the magnitude of a parcel's cross-stream motion.

Another characteristic of parcel's cross-stream motion in a meandering stream is illustrated in Fig. 4d: A large meander amplitude does not necessarily lead to strong lateral motion relative to the center. Large amplitude decaying meanders may have their spatial change in curvature canceled by the temporal change resulting in weak or no cross-stream motion. While on the other hand, small amplitude growing meanders may imply large lateral motions if its instantaneous change in amplitude is significant.

A second aspect of fluid motion examined in this study is the divergence pattern in the meandering stream. The introduction of a curvature-dependent ve- locity structure allows for changes in shear due to both conversion (i.e., from $f+\kappa_{s} u_{s}$ ) and distortion (i.e., changes of lateral scale). With the relaxation of the frozen field, the divergence pattern for a growing meander was established (Fig. 7) and found to be consistent with that of Bower's (1989). Further, the divergence is found to be curvature dependent. Large curvature in the stream path implies strong deviations from geostrophic balance and hence more pronounced divergence (or convergence) (Figs. 7 and 8). For the two opposite cases, that is, growing and decaying amplitude meanders, the patterns were also found to be opposite to each other. Divergence (or convergence) always takes place on the anticyclonic (or cyclonic) side upstream, and cyclonic (or anticyclonic) side downstream of growing crests (Fig. 9) and vice versa for decaying ones (Fig. 10). This striking change of divergence pattern can be easily understood in terms of simple physics that is pertinent to a meandering stream: As water parcels transit from trough to crest of a growing meander, centrifugal force tends to change from southward to northward (or rightward to leftward when facing downstream) and become increasingly strong in the latter direction due to increase in curvature in both time and space (along stream). Consequently, parcels are subject to lateral displacements along a density surface. For growing meanders, the temporal variation in curvature is strengthening the spatial 

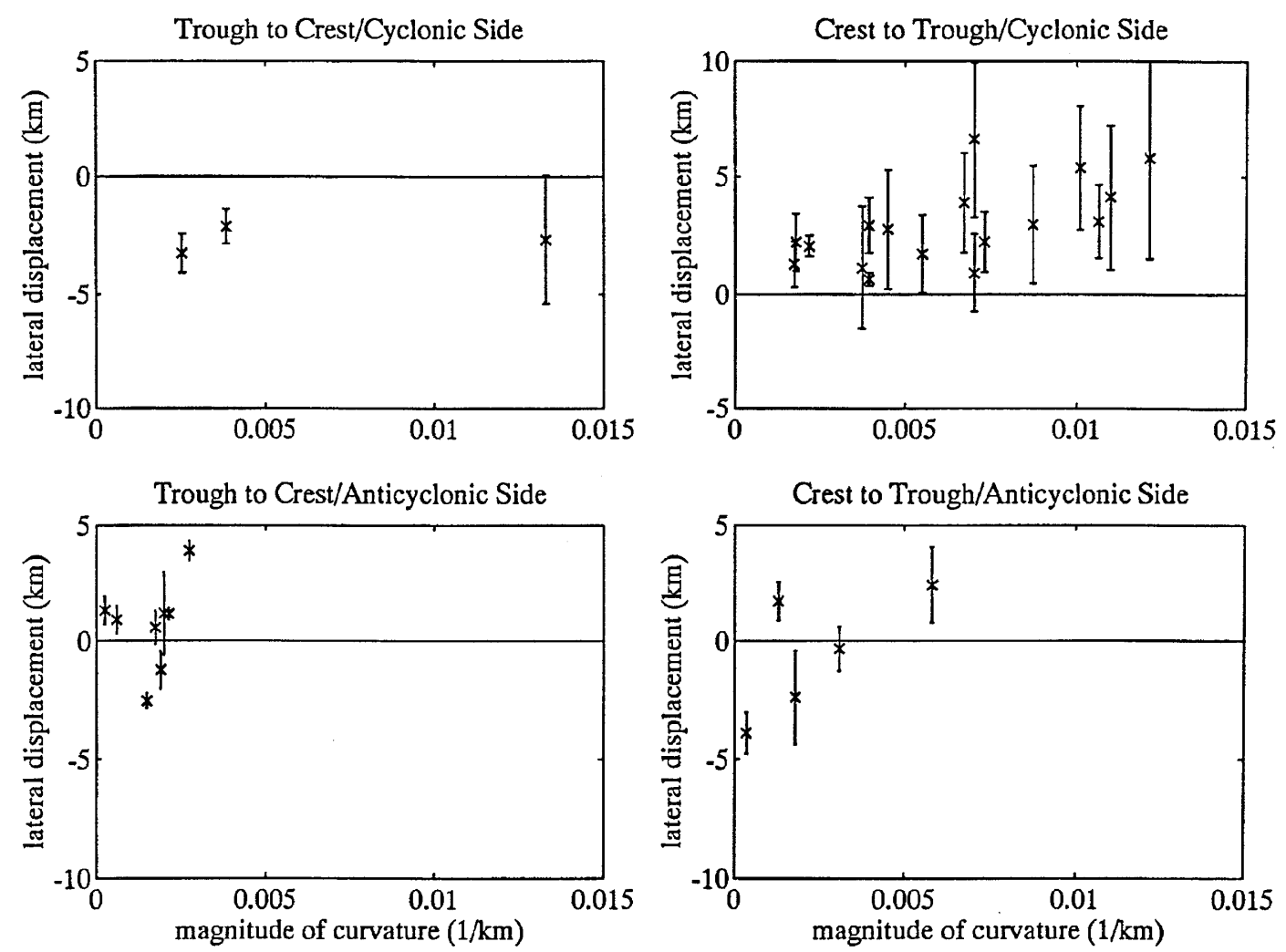

FIG. 10. As in Fig. 9 but for decaying meanders.

one and both contribute to determining the magnitude of cross-stream motion. Parcels at the center of the stream where velocity is larger are subject to a larger centrifugal force than those close to the edges and therefore result in a nonuniform cross-stream motion. This nonuniformity will in turn lead to horizontal divergence on the anticyclonic and convergence on the cyclonic side with the assumption that the alongstream variations

TABLE 2. Estimates of mean lateral displacement of water parcels in Gulf Stream meanders $\left(13-16^{\circ} \mathrm{C}\right)$.

\begin{tabular}{|c|c|c|c|c|}
\hline \multirow[b]{2}{*}{$\begin{array}{l}\text { Statis- } \\
\text { tics }\end{array}$} & \multicolumn{2}{|c|}{$\begin{array}{l}\text { Trough to crest } \\
\qquad(\mathrm{km})\end{array}$} & \multicolumn{2}{|c|}{$\begin{array}{l}\text { Crest to trough } \\
\qquad(\mathrm{km})\end{array}$} \\
\hline & Cyclonic & $\begin{array}{l}\text { Anti- } \\
\text { cyclonic }\end{array}$ & Cyclonic & $\begin{array}{l}\text { Anti- } \\
\text { cyclonic }\end{array}$ \\
\hline \multicolumn{5}{|c|}{ (a) Growing amplitude meanders } \\
\hline Mean & 1.628 & -0.745 & & 0.286 \\
\hline Std dev & 0.368 & 1.477 & & 1.132 \\
\hline Std error & 0.694 & 0.079 & & 0.068 \\
\hline$n$ & 4 & 55 & & 62 \\
\hline Conf level & $90 \%$ & $99 \%$ & & $99 \%$ \\
\hline \multicolumn{5}{|c|}{ (b) Decaying amplitude meanders } \\
\hline Mean & -2.625 & 0.444 & 1.351 & -0.656 \\
\hline Std dev & 0.678 & 2.241 & 1.009 & 2.736 \\
\hline Std error & 0.539 & 0.156 & 0.208 & 0.468 \\
\hline$n$ & 3 & 8 & 17 & 5 \\
\hline Conf level & $95 \%$ & $95 \%$ & $99 \%$ & $95 \%$ \\
\hline
\end{tabular}

a) Growing amplitude meander
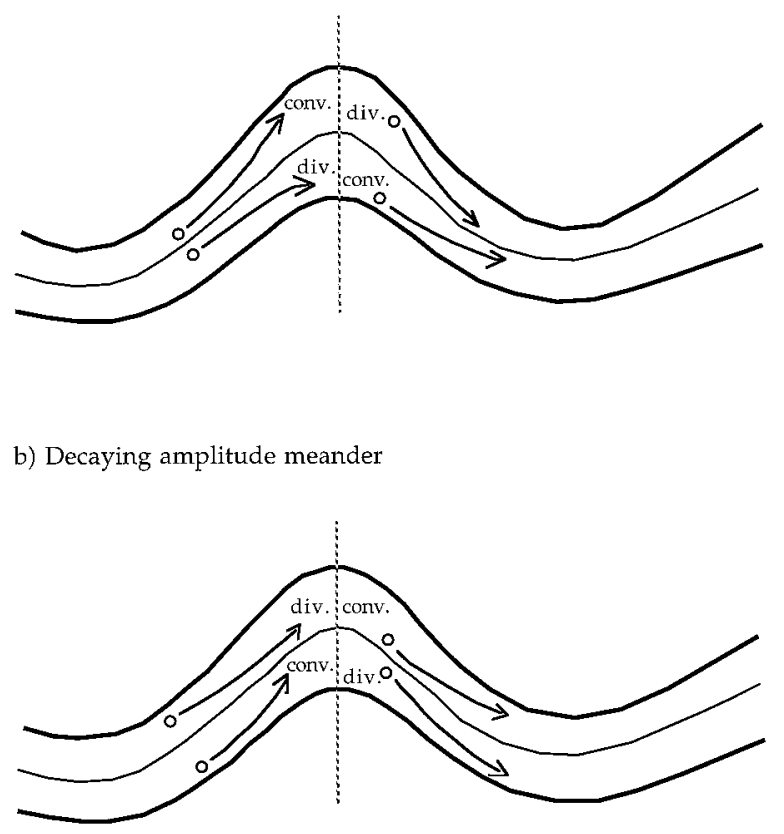

FIG. 11. Schematic view of a parcel's mean lateral displacement in growing and decaying meanders. 
of downstream velocity are negligible. The pattern switches sign when water parcels passing through a crest (or trough) where alongstream variation in curvature changes sign. On the other hand, if a meander decays in amplitude, parcels in the stream will tend to adjust themselves in response to the loss/decrease of the centrifugal force and therefore reverse the pattern established during the growing stage. Note that unlike the growing meander case where both the temporal and spatial changes have the same effect on a parcel, the temporal change in curvature is competing with the spatial change for decaying meanders. The magnitude of lateral motion will be determined by the net effect of the two opposing factors. Between the growing and decaying meander regimes, there can be a steady regime in which no changes in amplitude occur. However, the crossstream motion in this regime will not vanish because spatial change in curvature is still present. A zero lateral displacement of a parcel relative to the stream can only be expected in a decaying meander regime where temporal change in relative vorticity cancels out exactly the spatial (or advective) one. This argument may help to explain the situation of float 192 discussed in section 4 where little cross-stream displacement was observed while transiting through a decaying meander of $O(100$ $\mathrm{km})$ in amplitude.

One of the consequences of not distinguishing between growing and decaying meanders is the blurring out of the divergence pattern associated with decaying meanders. Only the pattern for growing meanders was observed as from Bower's analysis (1989) since these were more common in the area of that study. This can be clearly demonstrated by combining the corresponding panels in Figs. 7 and 8. The divergence pattern for growing meanders will persist but with an increase in scatter.

A final point that is worth noting from this study is the connection between the state of a meander and the behavior of individual fluid parcel. Growth and decay of meanders lead to different dynamical adjustments in velocity and density fields. Particles moving in such a stream are carrying information on the meandering state. The principal signature is the lateral shift of the locus of maximum downstream velocity (SRC), which was introduced in the present analysis of potential vorticity balance. As discussed in section 2, this property of lateral displacement was further used to determine the growth or decay of a meander associated with a particular float passage. Another signature, less obvious but noticeably related to the growth or decay of a meander, is that a parcel's lateral (or vertical) velocity does not always change sign at the very peak (bottom) of crests (troughs), but may occur before or after (see Figs. 4a and $4 \mathrm{c}$ ). The analysis here indicates that this reflects the growth or decay of a meander amplitude. In a zerogrowth meander, a parcel's lateral motion is only associated with the alongstream change in curvature. As that change vanishes at the peak (bottom) of crests (troughs), the parcel's lateral motion will change direction. The situation is different for growing meanders: A parcel will continue to move beyond the peak (bottom) point of crests (troughs) for the temporal change in curvature $\partial \kappa / \partial t$ still presents although the spatial change $\partial \kappa / \partial s$ doesn't. It is opposite for decaying meanders; that is, a change in sign of the cross-stream motion should be expected upstream of the meander extremum. The float observations and their comparisons with satellite IR imagery provide justification of our argument in some cases, while others could not be substantiated for various reasons. Since IR imagery is crucial for the determination of the peak and bottom points of a meander, cloudy conditions preclude the analysis of many cases. Also the growth or decay rate of a meander will determine, according to the above argument, the magnitude of the delay or advance of the sign change in cross-stream velocity. Hence, weak temporal change in amplitude will make the details of a parcel's motion undiscernible. And finally, the temporal resolution of satellite imagery is often inadequate making the signature less recognizable for fast propagating meanders.

Acknowledgments. We would like to acknowledge the support of Office of Naval Research under Contract N0014-90-J-1602. Special thanks go to Dr. R. Watts for his valuable comments and critiques on the earlier version of this paper and to J. Hummon, O. Sato, and E. Kearns for helpful discussions throughout this work. Very helpful suggestions by the reviewers are also gratefully acknowledged.

\section{REFERENCES}

Bane, J. M., L. M. O'Keefe, and D. R. Watts, 1989: Mesoscale eddies and submesoscale coherent vortices: Their existence near and interactions with the Gulf Stream. Mesoscale/Synoptic Coherent Structures in Geophysical Turbulence, J. C. J. Nihoul and B. M. Jamart, Eds., Elsevier Science, 501-518.

Bower, A. S., 1989: Potential vorticity balances and horizontal divergence along particle trajectories in Gulf Stream meanders east of Cape Hatteras. J. Phys. Oceanogr., 19, 1669-1681.

- and H. T. Rossby, 1989: Evidence of cross-frontal exchange processes in the Gulf Stream based on isopycnal RAFOS float data. J. Phys. Oceanogr., 19, 1177-1190.

Bryden, H. L., 1980: Geostrophic vorticity balance in midocean. $J$. Geophys. Res., 85, 2825-2828.

Chew, F., 1974: The turning process in meandering currents: A case study. J. Phys. Oceanogr., 4, 27-57.

Gilman, C. S., 1988: A study of the Gulf Stream downstream of Cape Hatteras 1975-1986. M.S. thesis, Graduate School of Oceanography, University of Rhode Island, $77 \mathrm{pp}$.

Halkin, D., and H. T. Rossby, 1985: The structure and transport of the Gulf Stream at $73^{\circ} \mathrm{W}$. J. Phys. Oceanogr., 15, 1439-1452.

Hall, M. M., 1986: Horizontal and vertical structure of the Gulf Stream velocity field at $68^{\circ} \mathrm{W}$. J. Phys. Oceanogr., 16, 18141828.

Holton, J. R., 1979: An Introduction to Dynamic Meteorology. 2d ed. Academic Press, 391 pp.

Hummon, J., 1995: A Gulf Stream-warm core ring interaction-Case study using statistical optimal interpretation. Ph.D dissertation, Graduate School of Oceanography, University of Rhode Island, 181 pp. 
Kontoyiannis, H. and D. R. Watts, 1994: Observations on the variability of the Gulf Stream path between $74^{\circ} \mathrm{W}$ and $70^{\circ} \mathrm{W}$. J. Phys. Oceanogr., 24, 1999-2013.

Leaman, K. D., E. Johns, and H. T. Rossby, 1989: The average distribution of volume transport and potential vorticity with temperature at three sections across the Gulf Stream. J. Phys. Oceanogr., 19, 36-51.

Lee, T., 1994: Variability of the Gulf Stream path observed from satellite infrared images. Ph.D. dissertation, Graduate School of Oceanography, University of Rhode Island, $188 \mathrm{pp}$.

Lindstrom, S. S. and D. R. Watts, 1994: Vertical motion in the Gulf Stream near $68^{\circ}$ W. J. Phys. Oceanogr., 24, 2321-2333.

Newton, C. W., 1959: Synoptic comparisons of jet stream and Gulf Stream systems. The Atmosphere and the Sea in Motion, B. Bolin, Ed., Rockefeller Inst. Press, 288-304.

- 1978: Fronts and wave disturbances in Gulf Stream and atmospheric jet stream. J. Geophys. Res., 83(C9), 4697-4706.
Palmén, E., and C. W. Newton, 1969: Atmospheric Circulation Systems. Academic Press, 603 pp.

Rossby, H. T., A. S. Bower, and P. T. Shaw, 1985: Particle pathways in the Gulf Stream. Bull. Amer. Meteor. Soc., 66, 1106-1110.

Shaw, P. T., and H. T. Rossby, 1984: Towards a Lagrangian description of the Gulf Stream. J. Phys. Oceanogr., 14, 528-540.

Song, T., H. T. Rossby, and E. Carter, 1995: Lagrangian studies of fluid exchange between the Gulf Stream and the surrounding waters. J. Phys. Oceanogr., 25, 46-63.

Tracey, K. L., and R. D. Watts, 1986: On Gulf Stream meander characteristics near Cape Hatteras. J. Geophys. Res., 91, 75877602.

Watts, D. R., and W. E. Johns, 1982: Gulf Stream meanders: Observations on propagation and growth. J. Geophys. Res., 87, 94679476. 